\title{
Apprendimento e insegnamento del «essico» di alta disponibilità
}

\author{
M ilena Bini \\ Almudena Pernas \\ M ercedes Sánchez
}

\section{Abstract}

Based on empirical data, this paper confirms the validity of Bini's hypothesis (in press) regarding the difficulties of learning lexis referring to everyday life. In addition to this, it al so proves that $L 1$ has an influence on the process of acquisition of these words together with L2 and hence producing hybridisms that crystalise.

\section{Introduzione}

II presente contributo concerne l'apprendimento e l'insegnamento delle parole di «alta disponibilità» che sono state reperite nell'inventario lessicale del Livello Soglia. L'indagine che presentiamo fa parte di un lavoro di più ampio respiro sull'apprendimento del lessico dell'italiano come lingua straniera.

Con questo studio ci proponiamo di dimostrare la validità dell'ipotesi circa la difficoltà di apprendimento, da parte di studenti stranieri, delle parole della vita quotidiana, definite di «alta disponibilità» perché, come afferma D e M auro (1980), sono ben note ai parlanti nativi pur essendo pronunciate o scritte piuttosto raramente. ${ }^{1}$ Q uest'ipotesi della difficoltà di apprendimento è stata formulata e sottoposta a verifica in uno studio precedente (Bini, in stampa) mediante un sondaggio su un campione di 51 parole anch'esse reperite nell'inventario lessicale del Livello Soglia. In generale i risultati del primo sondaggio parevano indicare che l'ipotesi fosse corretta. $\mathrm{M} \mathrm{a}$, poiché la difficoltà di apprendimento non sembrava uguale per tutte le parole, abbiamo voluto ampliare lo studio alle restanti 78 unità lessicali dello stesso tipo comprese nell'inventario suddetto.

O Itre a verificare la teoria della difficoltà di apprendimento, il nostro lavoro si prefigge un secondo obiettivo: mettere in luce alcuni effetti della vicinanza tipologica della L1 (lingua materna) e della L2 (lingua bersaglio) nell'acquisizione delle parole della vita quotidiana. 
1. Le parole di «alta disponibilità» costituiscono una fascia del vocabolario di base (VD B) dell'italiano elaborato da D e M auro nel 1980 e aggiornato nel 1989. Si tratta di termini legati ad esperienze, fatti e oggetti della vita di tutti i giorni e per questa ragione sono ben noti a chiunque (s'intende: parlanti nativi). Ciononostante, come dice De M auro, essendo fortemente legate al contesto, il loro uso non è frequente e per lo più è limitato alla lingua parlata. Si distinguono quindi dalle parole di alta frequenza per il loro basso rendimento. Per fare un esempio, nella fascia di parole di alta disponibilità troviamo termini che riguardano il tempo libero: prenotazione, intervallo, balletto, ferie; i mezzi di trasporto e i viaggi: abbonamento, passeggero, binari, dogana, coincidenza; la cucina e i cibi: pentola, coperchio, forchetta, griglia, salame; il clima: tuono, afa, piovoso; la salute: dentista, raffreddore, pillola; la scuola: iscrizione, bocciare, dettato ecc.

2. Per quanto riguarda l'insegnamento, sappiamo che queste parole compaiono nell'input a cui sono esposti i principianti perché le tendenze didattiche attuali stabiliscono come primo obiettivo lo sviluppo della competenza comunicativa in situazioni di vita quotidiana. $\mathrm{E}$ tutti i libri di testo di recente pubblicazione comprendono temi di questo genere. $\mathrm{N}$ ei livelli superiori, invece, argomenti come «a cucina», «la salute» $0 \ll i$ viaggi» vengono abbandonati o ripresi solo sporadicamente, al massimo a livello intermedio. D i conseguenza, la probabilità di occorrenza nell'input e le occasioni di usare parole come salame, raffreddore, abbonamento o pas seggero diminuiscono notevolmente, almeno in sede didattica, man mano che aumenta la competenza linguistica.

3. Le ricerche sull'acquisizione del lessico hanno evidenziato che una parola è ricordata facilmente se viene usata con frequenza a intervalli periodici, e meno facilmente se ripetuta in un breve lasso di tempo (Bahrick et alii, 1987: 344-349); ${ }^{2}$ ed inoltre che una parola si memorizza più facilmente nella misura in cui sorprende o colpisce l'immaginazione di chi la sente per la prima volta (C ook, 1991: 41). Q uindi, stando a queste teorie e tenendo conto delle caratteristiche e delle condizioni in cui compaiono nell'input, le parole di alta disponibilità hanno poche probabilità di entrare stabilmente nell'interlingua degli studenti stranieri. In primo luogo, perché vengono usate quasi esclusivamente a livello di principianti e non riprese a intervalli periodici (a differenza di quanto occorre con le parole di alta frequenza); in secondo luogo, perché le situazioni di vita quotidiana, a cui sono legate le parole considerate, sono per definizione situazioni di routine, arcinote agli studenti, e che di norma non presentano fatti 0 oggetti in grado di sorprenderli particolarmente 0 colpire la loro immaginazione. 
4. Ed ora prendiamo in esame il secondo obiettivo del nostro lavoro: mettere in luce gli effetti della vicinanza tipologica fra lo spagnolo e l'italiano nell'apprendimento del lessico della seconda lingua. Dalle ricerche sull'acquisizione di lingue affini pare assodato che, soprattutto nei casi di apprendimento spontaneo (non in sede scolastica), la percezione della vicinanza della L1 e della L2 favorisce il transfer e l'impiego della L1 come fonte di ipotesi per l'elaborazione dell'interlingua. In altre parole, quando gli apprendenti si rendono conto della somiglianza tra le due lingue, costruiscono la loro interlingua usando regole e parole della L 1, ma non tutte indistintamente e non senza una qualche trasformazione. Infatti le conoscenze della L1 interagiscono con i dati dell'input, con le conoscenze già acquisite della L2 e in sintonia con principi universali di acquisizione. ${ }^{3}$ Per quanto riguarda il lessico, Schmid (1994), autore di uno studio esaustivo sull'interlingua di immigranti spagnoli, ha fatto notare che il processo di apprendimento appena descritto dà come risultato un'interlingua con un'alta frequenza di ibridismi, vale a dire formazioni che combinano lessemi spagnoli con morfemi grammaticali italiani, e di elementi della L 1 con una differenza formale minima rispetto al lessema italiano. Q uesti fenomeni - osservati nell'interlingua degli immigranti - a nostro avviso hanno molte probabilità di verificarsi anche nell'interlingua degli studenti e soprattutto nella produzione di parole di alta disponibilità. Gli studenti infatti vengono a contatto con questo tipo di lessico prevalentemente mediantel'ascolto e le attività orali svolte in classe, situazione che riproduce in parte quella degli immigranti che apprendono ascoltando e parlando.

Considerando quindi a) le condizioni in cui gli studenti principianti vengono a contatto con le parole della vita quotidiana - soprattutto mediante il canale orale - e b) il fatto che i temi di questo tipo vengono poi abbandonati o ripresi solo sporadicamente a livello intermedio, abbiamo formulato la seguente ipotesi da verificare, in seguito, con i dati dello studio: nell'apprendimento del lessico di alta disponibilità gli studenti spagnoli a volte utilizzano le loro conoscenze della $L 1$, il che può dare come risultato la presenza nell'interlingua dei principianti, di ibridismi e persino di lessemi spagnoli se questi sono solo leggermente diversi dai corrispondenti lessemi italiani. Q ue sti ibridismi hanno molte probabilità di fossilizzarsi per cui potranno comparire anche a livello intermedio e avanzato.

\section{La rilevazione dei dati}

Per la verifica delle ipotesi abbiamo seguito la stessa procedura adottata nello studio precedente. II corpus, composto di 78 lessemi, appartiene all'inventa-

3. Sul tema del transfer cfr. Gass-Selinker (1983), Andersen (1983) e Kellerman (1983) fra gli altri. Per l'italiano cfr. Berretta (1986), Giacalone Ramat (1988), Bini (1991), Schmid (1994). 
rio lessicale del Livello Soglia, che complessivamente comprende 129 unità di alta disponibilità. Le altre 51 costituivano il corpus del primo studio.

La conoscenza delle parole è stata verificata mediante un test, da real izzare per iscritto, con foto e disegni degli oggetti a cui facevano riferimento le parole dello studio; per le parole astratte, per i verbi, e per i nomi di azione abbiamo preparato dei dialoghetti e delle frasi da completare. II test è diviso in 13 parti, ognuna con un argomento diverso: ozio, mezzi di trasporto, viaggi e vacanze, società e strutture sociopolitiche, cibi e bevande, servizi medici, clima, servizi pubblici, scuola, convenevoli, stati d'animo, materiali e qualità.

Al test sono stati sottoposti 158 studenti ispanofoni iscritti a un corso di italiano presso due sedi della Escuela O ficial de Idiomas di M adrid, una situata in centro e l'altra quasi in periferia. Lo scopo di prendere informanti da due sedi diverse della stessa scuola è stato quello di ottenere un campione diversificato. D ei 158 studenti, 53 erano principianti con un livello consolidato (110 ore di insegnamento), 44 erano di livello intermedio (200 ore), 37 di livello intermedio avanzato (290 ore) e 24 di livello avanzato (380 ore). La fascia di età era compresa tra i 18 e 50 anni e la maggior parte non superava i 35 anni.

\section{I risultati}

A grandi linee i dati di questo studio sono congruenti con quelli ottenuti nel sondaggio realizzato su 51 parole e paiono confermare l'ipotesi della difficoltà di apprendimento delle parole di alta disponibilità; infatti, come vedremo, solo una piccola parte di lessemi (il 29,6\%) pare essere conosciuta dalla maggior parte degli studenti. Però, come è accaduto nello studio precedente, i risultati indicano che non tutte le parole considerate entrano con eccessiva difficoltà nel vocabolario produttivo degli studenti spagnoli.

$D$ all'analisi delle occorrenze, disposte in ordine di frequenza e secondo i livelli di competenza - principiante, intermedio, intermedio-avanzato e avanzato - (Tabella 1) abbiamo riscontrato che i valori di frequenza delle 78 parole a livello avanzato (ultima colonna di destra) formano un continuum che inizia con la frequenza 0 del lessema passatempo e si chiude con il $100 \%$ della parola dentista. Un'analoga variazione dei valori può essere osservata anche agli altri livelli. T uttavia, I'ordine di frequenza delle parole ai primi 3 livelli non corrisponde esattamente a quello del livello avanzato.

All'interno del continuum si disegnano due fasce di parole abbastanza definite pur avendo dei confini labili. U na fascia si situa in testa alla lista, la seconda in coda. La prima fascia di 25 unità, pari al 32\% del campione, comprende le parole che vanno da passatempo a fornaio. Q ueste parole registrano valori di frequenza tendenzialmente molto bassi - non raggiungono il $50 \%$ a nessun livello - il che dimostra che la maggior parte degli studenti non le conosce. La seconda fascia, composta di 23 lessemi - da salame a dentista - 
pari al 29,6\% del campione, registra valori tendenzialmente alti: nella maggior parte dei casi supera il $50 \%$ e i valori aumentano progressivamente con il passaggio da un livello all'altro. Q ueste cifre indicano che le parole conside rate sono abbastanza note agli studenti. La fascia intermedia comprende 30 unità - da matematica a plastica - pari al 38,4\% del campione. Si tratta di una zona mista più sfumata, che non possiede delle caratteristiche così definite come le due fasce situate agli estremi. Comprende parole quasi sconosciute a livello di principianti che invece registrano valori abbastanza alti a livello avanzato, altre che hanno valori medi a quasi tutti i livelli, e altre ancora in cui le percentuali si alzano a livello intermedio per poi abbassarsi leggermente a livello avanzato.

Insomma, alcune parole presentano maggior difficoltà di altre. M a per quale ragione? La bassa frequenza nei nostri dati di alcuni termini può essere spiegata dalla possibilità che offre la lingua di usare termini al ternativi. Infatti compaiono proprietario e direttore al posto di principale; evidenziatore e segnalatore al posto di pennarello; ferry al posto di traghetto; lavorativi anziché feriali; stipendio anziché salario; panificio e forno anziché fornaio. $E$ ancora: aritmetica al posto di matematica; assassinio al posto di omicidio;, pastiglia e pasticca anziché pillola.

Altre volte la parola viene sostituita da una di significato simile. Infatti troviamo divertimento al posto di passatempo; incartare e impaccare al posto di imballare; pausa al posto di intervallo; pagamento anziché riscatto; umido anziché pi ovoso; rimandare anziché bocciare. E l'aggettivo insoddi ffatto viene sostituito da una lunga serie di altri aggettivi: infelice, dispiaciuto, deluso, depresso, amareggiato.

La maggior frequenza di alcune parole è invece meno facile da spiegare. Può darsi che gli studenti abbiano avuto più possibilità di usarle delle altre, malgrado non siano più frequenti delle altre nella lingua dei parlanti nativi. Ad esempio maiuscolo, divorziare o pittura, elencate fra le meno usate nel Lessico di frequenza dell'italiano parlato elaborato da D e M auro, hanno invece percentuali alte nei nostri dati. E si può inoltre ipotizzare che queste parole siano state memorizzate con più facilità delle altre perché hanno un corrispondente spagnolo uguale o simile. Infatti vi sono molte parole di questo tipo nella fascia di coda della lista, che comprende le parole più note agli studenti. Si tratta però di un'ipotesi da verificare con studenti di lingue materne diverse dallo spagnolo.

Vorremmo far notare comunque un dato interessante: nelle parole che paiono più note agli studenti si registrano percentuali piuttosto al te di ibridismi o produzioni devianti e questo fatto dimostra che anche se gli studenti le conoscono non sono entrate correttamente nel loro vocabolario produttivo.

Per quanto riguarda gli effetti della vicinanza tipologica osservati nella produzione delle parole considerate, i nostri dati confermano l'ipotesi che anche gli studenti, come gli immigranti, utilizzano le conoscenze della L 1 per costruire la loro interlingua. M a come le usano? Abbiamo rilevato diverse tendenze. 
T abella 1. 0 ccorrenze (percentuali) disposte in ordine di frequenza crescente in base ai risultati ottenuti a livello avanzato.

\begin{tabular}{|c|c|c|c|c|}
\hline Livello & $\begin{array}{l}\text { Principianti } \\
\text { (53) \% }\end{array}$ & $\begin{array}{l}\text { Intermedio } \\
\text { (44) \% }\end{array}$ & $\begin{array}{l}\text { Intermedio } \\
\text { avanzato (37) \% }\end{array}$ & $\begin{array}{l}\text { Avanzato } \\
\text { (24) \% }\end{array}$ \\
\hline Passatempo & - & - & - & - \\
\hline Principale & - & 2,2 & - & - \\
\hline Imballare & - & 2,2 & 2,7 & - \\
\hline Pennarello & - & 4,5 & 10,8 & 8,3 \\
\hline Intervallo & 3,7 & 6,8 & 16,2 & 12,5 \\
\hline Stampatello & 0,1 & 2,2 & 2,7 & 16,6 \\
\hline Espresso & - & 13,6 & 5,4 & 16,6 \\
\hline Centralino & - & 6,8 & 5,4 & 16,6 \\
\hline Prurito & - & 6,8 & 13,5 & 16,6 \\
\hline Trapanare & - & 2,2 & 5,4 & 16,6 \\
\hline Insoddisfatto & 0,1 & 13,6 & 13,5 & 16,6 \\
\hline Traghetto & - & 6,8 & 29,7 & 25,0 \\
\hline Coincidenza & 0,1 & 34,0 & 5,4 & 25,0 \\
\hline Riscatto & 13,2 & 31,8 & 35,1 & 33,3 \\
\hline Afa & 0,1 & 9,0 & 18,9 & 33,3 \\
\hline Iscrizione & 9,4 & 9,0 & 18,9 & 33,3 \\
\hline Trapano & - & 6,8 & 13,5 & 37,5 \\
\hline Tariffa & 15,0 & 36,3 & 32,4 & 37,5 \\
\hline Binario & 5,6 & 50,0 & 45,9 & 41,6 \\
\hline Tuono & 0,1 & 25,0 & 37,8 & 41,6 \\
\hline Roulotte & 24,5 & 27,2 & 32,4 & 45,8 \\
\hline Patente & 9,4 & 43,1 & 56,7 & 45,8 \\
\hline Feriali & - & 6,8 & 21,6 & 45,8 \\
\hline Salario & 12,2 & 29,5 & 45,9 & 45,8 \\
\hline Fornaio & 3,7 & 4,5 & 13,5 & 45,8 \\
\hline M atematica & 52,8 & 29,5 & 51,3 & 50,0 \\
\hline Piovoso & 24,5 & 27,2 & 37,8 & 50,0 \\
\hline Analisi & 62,2 & 75,0 & 78,3 & 50,0 \\
\hline Salumi & 5,6 & 2,2 & 27,0 & 50,0 \\
\hline D emocristiano & 13,2 & 22,7 & 18,9 & 50,0 \\
\hline Griglia & 0,1 & 15,9 & 27,0 & 54,1 \\
\hline O micidio & 0,1 & 31,8 & 54,0 & 54,1 \\
\hline Trasparente & 26,4 & 43,1 & 54,0 & 58,3 \\
\hline Abbonamento & 9,4 & 22,7 & 54,0 & 62,5 \\
\hline Traduzione & 24,5 & 45,4 & 54,0 & 62,5 \\
\hline Paziente & 20,7 & 27,2 & 27,0 & 66,6 \\
\hline Pillola & - & 27,2 & 45,9 & 66,6 \\
\hline Vocabolario & 7,5 & 20,4 & 59,4 & 66,6 \\
\hline Spiccioli & 0,1 & 40,9 & 45,7 & 66,6 \\
\hline
\end{tabular}


T abella 1.

\begin{tabular}{|c|c|c|c|c|}
\hline Livello & $\begin{array}{l}\text { Principianti } \\
\text { (53) \% }\end{array}$ & $\begin{array}{l}\text { Intermedio } \\
\text { (44) \% }\end{array}$ & $\begin{array}{l}\text { Intermedio } \\
\text { avanzato (37) \% }\end{array}$ & $\begin{array}{l}\text { Avanzato } \\
(24) \%\end{array}$ \\
\hline M ensa & 9,4 & 25,0 & 54,0 & 66,6 \\
\hline Prenotare & 30,1 & 68,1 & 67,5 & 66,6 \\
\hline Prenotazione & 30,1 & 63,6 & 56,7 & 70,8 \\
\hline N uoto & 18,8 & 38,6 & 45,9 & 70,8 \\
\hline Zanzara & 9,4 & 27,2 & 51,3 & 70,8 \\
\hline Tosse & 15,0 & 40,9 & 59,4 & 70,8 \\
\hline Signorina & 67,9 & 77,2 & 67,5 & 70,8 \\
\hline Bocciare & 11,3 & 36,3 & 56,7 & 75,0 \\
\hline Dizionario & 49,0 & 81,8 & 81,0 & 75,0 \\
\hline Ferie & 15,0 & 25,0 & 56,7 & 75,0 \\
\hline Condoglianze & 35,8 & 65,9 & 64,8 & 79,1 \\
\hline Tossire & 15,0 & 43,1 & 48,6 & 79,1 \\
\hline Balletto & 7,5 & 18,1 & 29,7 & 79,1 \\
\hline Elettricità & 64,1 & 63,6 & 56,7 & 79,1 \\
\hline Pigro & 5,6 & 31,8 & 54,0 & 79,1 \\
\hline Plastica & 37,7 & 50,0 & 64,8 & 79,1 \\
\hline Salame & 41,5 & 45,4 & 62,1 & 83,3 \\
\hline Scultura & 35,8 & 75,0 & 67,5 & 83,3 \\
\hline Comico & 73,5 & 63,6 & 83,7 & 83,3 \\
\hline T elegrafo & 47,1 & 59,0 & 70,2 & 83,3 \\
\hline Parentesi & 45,2 & 72,7 & 70,2 & 83,3 \\
\hline Virgola & 37,7 & 72,7 & 83,7 & 83,3 \\
\hline Passeggero & 15,0 & 52,2 & 56,7 & 87,5 \\
\hline Toilette & 32,0 & 52,2 & 54,0 & 87,5 \\
\hline Sindacato & 62,2 & 52,2 & 78,3 & 87,5 \\
\hline D ogana & - & 31,8 & 48,6 & 87,5 \\
\hline Terrorismo & 60,3 & 68,1 & 91,8 & 87,5 \\
\hline Ricetta & 62,2 & 81,8 & 89,1 & 87,5 \\
\hline D ettato & 22,6 & 45,4 & 56,7 & 87,5 \\
\hline Zero & 41,5 & 63,6 & 78,3 & 91,6 \\
\hline Ambasciata & 56,6 & 81,8 & 70,2 & 91,6 \\
\hline D entifricio & 24,5 & 34,0 & 72,9 & 91,6 \\
\hline Pittura & 33,9 & 72,4 & 72,9 & 91,6 \\
\hline Raffreddore & 5,6 & 45,4 & 70,2 & 91,6 \\
\hline Divorziare & 49,0 & 70,4 & 81,0 & 95,8 \\
\hline M aiuscolo & 50,9 & 56,8 & 70,2 & 95,8 \\
\hline Cantante & 83,0 & 77,2 & 81,0 & 95,8 \\
\hline Grazie & 88,6 & 88,6 & 91,8 & 95,8 \\
\hline D entista & 58,4 & 81,8 & 83,7 & 100,0 \\
\hline
\end{tabular}


1. La tendenza a mantenere la vocale spagnola quando una parola, esistente in tutte e due le lingue, si distingue per quell'unica vocale. Ad esempio compare recetta (ricetta) o vocabulario (vocabolario). E questo succede non solo se le due vocali sono vicine in quanto a grado di apertura, ma anche quando sono lontane come in sindicato (sindacato). Q uesto fenomeno può avere due spiegazioni: a) gli studenti hanno prodotto la parola spagnola ipotizzando che la parola fosse uguale anche in italiano; oppure b) conoscono la parola italiana ma il fatto di averla appresa dal parlato non ha consentito loro di notare la differenza di vocale che in queste parole è una vocale atona.

2. Un'altra tendenza è quella di inserire un gruppo consonantico della L1 estraneo alla L2. Ad esempio, pictura (pittura).

3. Abbiamo poi la tendenza ad usare la grafia spagnola al posto di quella italiana per rappresentare fonemi marcati della $L 2$ e inesistenti nella $L 1$ come «zeta»e «vu». Ad esempio, cero (zero), paciente (paziente), di vorciare (divorziare), e gracie (grazie) sono scritte con la «c», diccionario e dicionario (dizionario) con una e con due «c» e birgola (virgola) con la «b».

L'uso della grafia spagnola pare indicare che gli studenti abbiamo memorizzato le parole con il fonema della L 1 (spagnolo). I nfatti, abbiamo spesso produzioni di questo tipo a tutti i livelli compreso quello avanzato.

4. Vi sono inoltre ibridismi che paiono elaborati sulla base di un lessema spagnolo con l'aggiunta di un morfema italiano.

Ad esempio condolenze (condoglianze) da «condolen (cias) +ze».

$\mathrm{N}$ ella combinazione di lessemi spagnoli e morfemi italiani si aggiunge a volte una seconda tendenza: la generalizzazione della corrispondenza fra un elemento spagnolo e uno italiano (Schmid, 1994). Ad es. nella costruzione della parola truono (tuono) gli studenti hanno percepito che il dittongo «ue» dello spagnolo spesso viene reso con «uo» in italiano (come in «bueno» «buono», «fuego» «fuoco») ed hanno generalizzato questa corrispondenza. $E$ in embassata hanno generalizzato la corrispondenza di «j» in spagnolo e «ss» in italiano su modello di «tejido» «tessuto» e «bajo» «basso».

5. La presenza di un elemento spagnolo in parole italiane è un altro fenomeno ricorrente. Ad es. in draghetto (traghetto) o nuodo (nuoto) si può ipotizzare che gli studenti abbiano percepito la consonante più diffusa in spagnolo della coppia (sonora/ sorda) invece di quella corretta oppure che nel dubbio abbiano scelto la soluzione spagnola. É presente pero anche la tendenza contraria, vale a dire la scelta della soluzione più lontana dalla L1 per rendere suoni esistenti in tutte e due le lingue. È il caso di maggioscole e maioscole (maiuscole) in cui il dittongo «iu» viene sentito come un suono spagnolo e non italiano. 
6. Da ultimo dobbiamo far riferimento a un gruppo di produzioni nelle quali compare più di un fenomeno deviante e che, come nei casi precedenti, sembrano il risultato dell'impiego delle conoscenze della L1 più quelle della L2. Ad esempio piuvioso (it. piovoso, sp. lluvioso), alcune varianti di «ambasciata»: ambassada, embascada, imbassata e alcune varianti di «dentifricio»: dentrificio, dentrifico.

\section{Conclusioni e implicazioni didattiche}

I risultati dell'indagine hanno confermato le ipotesi iniziali: le parole di alta disponibilità entrano con difficoltà nel vocabolario produttivo degli studenti e la L1 interviene insieme alla L2 nell'apprendimento di queste parole dando come risultati ibridismi che si fossilizzano.

Per quanto riguarda l'insegnamento, lo studio ha messo in luce la necessità di riprendere i temi della vita quotidiana a intervalli periodici e di aiutare gli studenti a memorizzare le parole considerate con la pronuncia e la grafia corrette. Si dovranno quindi aumentare le attività di ascolto e di produzione orale su temi della vita di tutti i giorni e aggiungere a queste delle attività di produzione scritta guidata, per attirare l'attenzione degli studenti sulla grafia delle parole. Ad esempio, il completamento di trascrizioni di registrazioni autentiche da realizzare durante l'ascolto.

Le implicazioni didattiche dello studio riguardano anche la fase di valutazione. I risultati indicano infatti che, nella determinazione del livello di competenza degli studenti, il lessico di alta disponibilità va usato con cautela.

\section{Bibliografia}

AN DERSEN, R.W. (1983). «T ransfer to somewhere». In G ass-Selinker: 177-204.

BAHRICK, H.P.; PHELPS, E. (1987). «Retention of Spanish vocabulary over eight years». . . Exp. Psychol.: Learning, M emory and Cognition, XIII/2: 344-349.

BerRETTA, M. (1986). «Per uno studio dell'apprendimento del' 'italiano in contesto naturale: il caso dei pronomi personali atoni». In Giacalone Ramat: 329-352.

BINI, M . (1991). El italiano como segunda lengua: la adquis ción de los pronombrespersonales en ámbito escolar. M adrid: Editorial de la U niversidad Complutense.

- (in stampa). «Le parole di "alta disponibilità" nell'italiano lingua straniera».

Calvi, M .V. (1995). Didattica delle lingue affini. Spagnolo e italiano. M ilano: Guerini.

Cook, V. (1991). Second Language Learning and Language Teaching. Londra: Edward Arnold.

D e M AURo, T. (1989). 10 ed. Guida all'uso delle parole. Roma: Editori Riuniti.

- (1994). Come parlano gli italiani. Firenze: La N uova Italia.

De MaURo, T. et alii. (1993). Lessico di frequenza dell'italiano parlato. Milano: Etaslibri.

Galli de' Pratesi, N . (1981). Livello soglia. Strasburgo: Consiglio d'Europa. 
GaSS, S.; SelinkER, L. (1983). Language Transfer in Language Learning. Rowley $M$ ass.: N ewbury H ouse.

Gensinı, S.; Ved o Velli, M. Teoria epratica del glotto-kit. M ilano: Franco Angeli.

Giacalone Ramat, A. (1988). L'italiano tra le altre lingue: strategie di acquisizione. Bologna: Il Mulino.

- (1986). L'apprendimento spontaneo di una seconda lingua. Bologna: II M ulino: 329-352.

Kellerman, E. (1983). «N ow you see it, now you don't». In G as- Selinker: 112-134. SCH MID, S. (1994). L'italiano degli spagnoli. M ilano: Franco Angeli.

VED O VELLI, M . (1983). «Parametri di conoscenza ricettiva di vocabolario di base». In Gensini-Vedovelli: 179-196.

- (1993). «Confronti fra il LIP e le altre liste di frequenza dell'italiano». In De M auro et alii (1993): 119-147. 